\title{
Vom deutschen Gehilfenverein zur DNSAP Die Entwicklung einer Partei im Überblick
}

\author{
Early National Socialists in Austria-Hungary \\ An Overview of the Development of the 'German National Socialist Workers' Party'
}

While the details of the rise of the national socialists of Hitler's NSDAP in Germany are widely known, the ideological roots of this movement and its origin in the Austro-Hungarian empire are often left undiscussed. This paper focuses on the ideological development of what later would become the national socialist party of Austria and the relation of this party to Hitler's NSDAP and the Czechoslovakian national socialists.

Keywords: Austria-Hungary - DNSAP - national socialism - NSDAP

\section{Einleitung}

Die Parteigeschichte der in (Deutsch-)Österreich tätigen Deutschen Nationalsozialistischen Arbeiterpartei (DNSAP) ist weit weniger aufgearbeitet als jene der mehr oder weniger zeitgleich in Deutschland aktiven NSDAP - dies vermutlich auch aufgrund ihrer geringen Bedeutung in der politischen Landschaft Österreichs. Die ideologischen Wurzeln der DNSAP lassen sich allerdings über ihre Gründung 1918 hinaus zurückverfolgen. Die Entwicklungsgeschichte dieses Arbeitervereins, der sich von seinen deutschnationalen, sozialistischen Wurzeln hin zu einer nationalsozialistischen Partei entwickelte, soll das Thema dieses Beitrags sein.

Die Aufarbeitung der frühen Parteigeschichte gestaltet sich vor allem deswegen als schwierig, da sie nur spärlich dokumentiert ist. Die vorhandene Literatur, die auch diesem Beitrag zu gewissen Teilen zu Grunde liegt, wurde vorwiegend

\footnotetext{
${ }^{1}$ Natürlich gibt es zu dieser Thematik auch eine Reihe an tschechischer Literatur, die aufgrund mangelnder Sprachkenntnisse der Autorin nicht in den Aufsatz übernommen werden konnte. Es sei hingewiesen auf: MALíř, Politické strany. Die von den tschechoslowaki-
}

von den zeitgenössischen Parteimitgliedern verfasst und ist daher dementsprechend kritisch zu betrachten. ${ }^{1}$ Von der weiteren Darstellung ab der Auflösung der Partei 1933 bzw. 1934 wurde hingegen abgesehen, da hierzu bereits ausführliche Literatur ${ }^{2}$ existiert.

Eine vereinsrechtliche Untersuchung soll in diesem Beitrag nur in groben Grundzügen erfolgen. Kern des Beitrages ist die ideologische Entwicklung der Partei auf Grundlage der verschiedenen Parteiprogramme.

\section{Zur Rechtsnatur}

Aufgrund der unklaren Rechtsnatur der Gehilfen,,vereine“ und „Parteien“, die im Zuge dieses Beitrags behandelt werden, ist es angebracht, die rechtlichen Rahmenbedingungen zuerst und gesondert zu betrachten. Zunächst gestaltet sich die Suche nach Dokumenten, die die eindeutige

schen Parteimitgliedern publizierten Werke, die in diesem Beitrag verwendet wurden, sind alle in deutscher Sprache verfasst.

2 Siehe u.a. ReITER-ZATLOUKAL, ROTHLÄNDER, SCHÖLNBERGER, Österreich 1933-1938; TALOS, NeUGEBAUER, Austrofaschismus. 
Rechtsnatur der Vereine und Parteien belegen, als schwierig. Grund dafür ist, dass die Quellen nicht klar erkennen lassen, ob die frühen Gehilfenvereine in Böhmen und Mähren tatsächlich Vereine im rechtlichen Sinn waren, oder ob sie vielmehr Treffpunkte für lokale Arbeiter („Stammtische“) waren, die keine Rechtspersönlichkeit hatten.

Sollte es sich um Vereine i.S.d. Vereinsgesetzes 1867 gehandelt haben, so gab es zwei Möglichkeiten. Zum einen konnten sich die Mitglieder als nichtpolitischer Verein statuieren, zum anderen als politischer Verein nach $\S \S 29-35$ leg.cit.

Eine Gründung der Gehilfen,,vereine“ als politische Vereine ist unwahrscheinlich, da politische Vereine nach $\S 33$ des Vereinsgesetzes keine Zweigvereine gründen, Verbände schließen oder untereinander durch schriftlichen Verkehr oder Abgeordnete kommunizieren durften. Auch war es einem politischen Verein bei Strafe untersagt, mit einem nicht politischen Verein in Verbindung zu treten. ${ }^{3}$ Dass zwischen den einzelnen Gehilfenvereinen und den daraus später entstandenen Parteien reger Austausch, auch durch Entsenden von Rednern, herrschte, ist aber hinreichend belegt. ${ }^{4}$

Wahrscheinlicher ist, dass die frühen Gehilfenvereine und ihre Nachfolger, auf die unten näher eingegangen wird, als nicht politische Vereine auf Grundlage des Vereinsgesetzes von 1867 gegründet wurden. Klarheit könnte aber nur ein Blick in die Statuten - sofern diese noch existieren - bringen. Dazu wären Nachforschungen in den

\footnotetext{
${ }^{3}$ MAYRHOFER, Handbuch für den politischen Verwaltungsdienst 2, 120.

${ }^{4}$ Dazu ausführlicher im entsprechenden Teil des Aufsatzes.

${ }^{5}$ Nach Auskunft der Vereinsbehörde vernichtet sie alle Aufzeichnungen über Vereine, die länger als zehn Jahre aufgelöst sind. Dies inkludiert den gesamten Bestand aus der Monarchie, sofern die Akten nicht vom Stadt- und Landesarchiv Wien gerettet wurden. Vereinzelte Dokumente zur DNSAP, die die Gauleitung ab 1939 gesammelt hatte, sind im Wiener Stadt- und Landesarchiv zu finden, betreffen aber nicht die frühen
}

örtlich in Frage kommenden Archiven nötig. Eine Anfrage bei der Wiener Vereinsbehörde blieb erfolglos. ${ }^{5}$ Da das Vereinsgesetz 1867 von der provisorischen Nationalversammlung in die Republik Deutschösterreich übernommen wurde, ist davon auszugehen, dass es in Monarchie und Republik die Rechtsgrundlage für die - 1903 gegründete und 1918 in DNSAP umbenannte - Arbeiterpartei war. Über die genaue Rechtsgrundlage der Vereine, die von der Monarchie übergeleitet wurden, wurde bis 1929 eingehend diskutiert. ${ }^{6}$ Dass sich die Bezeichnung der Gruppierung von „Verein" in „Partei“ geändert hat, ist wohl auf die Einführung des allgemeinen (Männer-)Wahlrechts in der Monarchie zurückzuführen. ${ }^{7}$ Schon die Reichsratswahlordnung vom 26. Jänner 1907 spricht in ihrem $\S 23$ Abs. 3 und 4 von „wahlwerbenden Parteien“. Um eine Partei i.S.d. Parteiengesetzes handelte es sich hierbei nicht. Anstatt den Anhang „Partei“ als Hinweis auf die Rechtssubjektivität zu werten, ist es wahrscheinlich zutreffender, dass die Vereine damit zum Ausdruck bringen wollten, dass sie als wahlwerbende Partei an den Wahlen teilzunehmen gedachten.

Die genaue Rechtslage dieser „politischen Parteien" blieb weitestgehend unklar und war auch noch in der 1. Republik Gegenstand des wissenschaftlichen Diskurses. „Aus den Wahlordnungen geht an mehreren Stellen klar hervor, daß die Wahlpartei mit der politischen Partei nicht identisch ist“. ${ }^{8}$ Es ist für eine „politische Partei“ wenig sinnvoll, zumindest aber für die Beteiligung an

Gruppierungen der deutschen nationalen Sozialisten. Näheres zur Bezeichnung der Gruppierung im frühen Stadium als nationale Sozialisten: siehe Anm. 22.

${ }^{6}$ U.a. LENHOFF, Die politische Partei als Rechtssubjekt; als Antwort darauf: KRAUS, Die politische Partei als Rechtssubjekt.

${ }^{7}$ Zuerst die Badenische Wahlrechtsreform von 1896, die eine fünfte Kurie mit einem Wahlrecht für Männer ab dem 24. Lebensjahr einführte, danach die Beck'sche Wahlrechtsreform von 1907, die ein allgemeines Männerwahlrecht schuf.

${ }^{8}$ KRAUS, Die politische Partei als Rechtssubjekt 495. 
Wahlen nicht zwingend, sich als politischer Verein i.S.d. §§ 29-35 Vereinsgesetzes 1867 zu statuieren. Dementsprechend wird es sich bei der 1903 gegründeten DAP um einen Verein i.S.d. Vereinsgesetzes, und nicht um einen politischen Verein i.S.d. §§ 29-3leg.cit. gehandelt haben.

Mangels vorhandener Statuten werden die folgend behandelten "Vereine" als Rechtssubjekte i.S.d. Vereinsgesetzes 1867 betrachtet, nicht jedoch als politische Vereine. $\mathrm{Ob}$ einige davon $\mathrm{Zu}-$ sammenschlüsse ohne Rechtsgrundlage und Rechtssubjektivität waren, kann aus den angeführten Gründen nicht sicher belegt werden. Der folgende Beitrag widmet sich daher nicht dem Vereinsrecht, sondern der ideologischen Entwicklung jener Partei, die eine Entwicklung von einer national sozialistischen Gesinnung hin zu einer nationalsozialistischen vollzog und ab den 1920er Jahren kontinuierlich dem Einfluss Hitlers unterlag.

\section{Die ideologischen Wurzeln}

Literatur und Zeitgenossen sind sich nicht ganz einig, ob die Geschichte der späteren DNSAP beim - vorwiegend in Böhmen und Mähren aktiven - Deutschen Gehilfenverein, der 1885 gegründet wurde, beginnt, oder beim Linzer Programm von 1882; es sind sich jedoch alle einig, dass die ideologischen Wurzeln bei dem Verfasser des Linzer Programmes, Georg von Schönerer, $\mathrm{zu}$ finden sind. ${ }^{9}$

Schönerer, der als Abgeordneter zuerst für die liberale deutsche Fortschrittspartei in den Reichsrat gewählt worden war, sich von dieser aber bald ideologisch entfernte, gründete im Juni 1882 den „Deutschnationalen Verein“. Auch wenn im Statut des Vereins selbst vorerst keine explizit antisemitischen Forderungen niedergeschrieben

\footnotetext{
${ }^{9}$ BERNER, Vom Gesellenverein zum nationalen Sozialismus 8 .

${ }^{10}$ PICHL, Georg Ritter von Schönerer 100.

${ }^{11}$ WLADIKA, Hitlers Vätergeneration 151.
}

waren, so stand der Verein nur "deutschen" Männern offen. Da „der Jude kein Deutscher"10 sei, kann aber von einer antisemitischen Haltung des Vereins ausgegangen werden. So teilte man auch Viktor Adler, als dieser dem Verein beitreten wollte, mit, dass seine Mitgliedschaft unerwünscht sei. ${ }^{11}$

Im September desselben Jahres wurde das „Linzer Programm der Deutschnationalen“ veröffentlicht. Das Programm stützte sich auf die demokratischen Forderungen von 1848, das Parteiprogramm des Historikers Heinrich Friedjung von 1880 und ein Parteiprogramm, das Schönerer selbst bereits 1879 entworfen hatte. ${ }^{12}$ Das Linzer Programm verlangte unter anderem eine Personalunion mit Ungarn, die ausschließliche Verwendung der deutschen Sprache beim Heer, in öffentlichen Ämtern und Protokollen, in der Volks- und Mittelschule sowie bei sämtlichen Staatsprüfungen und Rigorosen. Zwar forderte das Programm neben Vereins- und Pressefreiheit auch ein direktes Wahlrecht, jedoch sollte ein passives Wahlrecht nur „deutschen Männern“ zukommen. Ein klarer sozialreformatorischer Standpunkt trat vor allem bei den Forderungen nach einer Reform der Gewerbeordnung, beim Arbeitnehmerschutz (u.a. Einschränkung der Kinder- und Frauenarbeit sowie Festsetzung einer Normalarbeitszeit) und bei steuerlichen Forderungen zutage. Mit dem Deutschen Reich forderte man ein dauerhaftes (staatsvertragliches) Bündnis sowie eine Zollunion. Antisemitische Forderungen, wie sie im Programm von Schönerer und jenem von Friedjung zu lesen sind, waren 1882 im Linzer Programm nicht enthalten. Dies

12 Ebd. 152; Die Programme sind nachzulesen bei BERCHTOLD, Österreichische Parteiprogramme 18681966. 
ist wohl auf die Mitarbeit Viktor Adlers ${ }^{13}$ am Programm zurückzuführen, der maßgeblich an der Entwicklung - allen voran der sozialistischen Teile des Programms - beteiligt war, bevor man ihm erklärte, dass er unerwünscht sei. Nach 1885 fügte man dem Linzer Programm jedoch einen zwölften Punkt hinzu: „Zur Durchführung der angestrebten Reformen ist die Beseitigung jüdischen Einflusses auf allen Gebieten des öffentlichen Lebens unerläßlich. “14

Das Linzer Programm war wesentlich gemäßigter als das Programm von Schönerer aus dem Jahr 1879 und das Programm von Friedjung. Beide Programme enthielten neben antisemitischen Forderungen auch die Trennung von Kirche und Staat, dies vor allem im schulischen Bereich. Interessant ist auch, dass im Linzer Programm eine Forderung fehlt, die im Aufruf zur Gründung der deutschnationalen Partei 1881 enthalten ist, nämlich die Einführung einer obligatorischen Zivilehe. ${ }^{15}$

Beinahe zeitgleich, 1885, wurde der erste der Deutschen Gehilfenvereine im südböhmischen Budweis [České Budějovice] gegründet. Gleichnamige Vereine waren bald besonders in Böhmen und Mähren weit verbreitet, sodass es im Jahre 1888, am 4. November, in Reichenberg bereits einen „Deutschen Gehilfentag für Böhmen und Mähren" gab. Schon dort war die Abneigung gegen die tschechische Arbeiterschaft, die sich durch die Parteiprogramme bis hin zum Programm der 1918 gegründeten DNSAP zieht, klar erkennbar: „Die tschechischen Arbeiter werden bevorzugt, weil dieselben billiger arbeiten und sich, da sie gar keine Standesehre besitzen, die

\footnotetext{
${ }^{13}$ WLADIKA, Hitlers Vätergeneration 152, 155. Dass Adler für den Deutschnationalen Verein und Schönerer tätig war, führt Wladika auf einen antisemitischen "Grundkonsens" in Form eines „wirtschaftlichen Antisemitismus“ Adlers zurück. Der von Schönerer vertretene rassische Antisemitismus führte schließlich dazu, dass Adler die Aufnahme in den Deutschnationalen Verein verweigert wurde. Er trat daraufhin bekanntlich den Sozialdemokraten bei. Ausführlich hierzu: WLADIKA, Hitlers Vätergeneration $156 f$.
}

niederträchtigste Behandlung gefallen lassen." 16 Im Zuge der Zusammenkunft wurden auch die Vertreter der (sozialdemokratischen) Arbeiterpartei in Wien kritisiert, da diese „die Verhältnisse in Böhmen und Mähren nicht kennen und nicht wissen, daß hier der nationale Standpunkt auch der wirtschaftliche Standpunkt ist" ${ }^{\prime 1}{ }^{17} \mathrm{Ne}-$ ben ihrem Ziel, der „Tschechisierung des deutschen Handwerks und Gewerbes Einhalt zu tun“18, das sie vor allem durch Arbeitsvermittlung zu erreichen versuchten, stellten die Gehilfenvereine Unterstützungskassen und Bibliotheken für ihre Mitglieder zur Verfügung. Letztere dienten der Fortbildung der Arbeiterschaft in einer Zeit, in der frei zugängliche Bibliotheken noch eine Seltenheit waren.

Ideologisch setzen sich zwar sowohl Schönerer als auch der Deutsche Gehilfenverein für die Interessen der Arbeiter ein, jedoch auf verschiedenen Ebenen. Während Schönerer, wie schon am Parteiprogramm ersichtlich, weitreichende rechtliche und staatliche Reformen forderte, die bis in die Tiefen der Verwaltung, der Diplomatie und der Gesetze der Monarchie reichten, waren die Gehilfenvereine ein Netzwerk aus lokalen Vereinen, das sich primär gegen die tschechischen Arbeiter und für die Förderung der deutschen Arbeiter einsetzte. Ihr Fokus lag auf Arbeitsvermittlung und Fortbildung ihrer Mitglieder. ${ }^{19}$ Es ist nicht davon auszugehen, dass sich die Mitglieder der frühen Gehilfenvereine über ihre lokalen Bedürfnisse hin-

${ }^{14}$ BERCHTOLD, Österreichische Parteiprogramme 18681966, 203.

15 Ebd. 192.

${ }^{16}$ BERNER, Vom Gesellenverein zum nationalen Sozialismus 9.

${ }^{17}$ Ebd. 10.

${ }^{18} \mathrm{KNIRSCH}$, Arbeiterbewegung 12.

${ }^{19}$ Ebd. 12; POKORNÝ, Vereine und Parteien in Böhmen 694. 
aus für die politischen Strömungen in der Monarchie einsetzten. ${ }^{20}$ Diese Vermutung bestätigt sich auch dadurch, dass die vielen Gehilfenvereine es trotz ihres gleichen Zieles aufgrund interner Differenzen für viele Jahre nicht schafften, einen Dachverband zu gründen. ${ }^{21}$ Für Hans Knirsch, den späteren Führer der tschechoslowakischen DNSAP, waren die „nicht politischen Arbeitervereine auch Stütz- und Sammelpunkte für den politischen Kampf".22 Viel wahrscheinlicher als eine politische Tätigkeit der Vereine ist, dass Anhänger national sozialistischer Bewegungen die lokalen Vereine als Plattformen nutzten, um ihre Ideologie zu verbreiten und Wählerstimmen unter den Arbeitern zu gewinnen. ${ }^{23}$

Die ideologische Kluft zwischen den sozialdemokratisch gesinnten Vertretern der Arbeiter in den österreichischen Teilen der Monarchie, welche die Lage aller Arbeiter zu verbessern suchten, und den deutschen Arbeitervereinen Böhmens und Mährens, die lediglich an der Verbesserung der Verhältnisse der deutschen Arbeiter interessiert waren, führte letztendlich zu einer völligen

\footnotetext{
${ }^{20}$ Dies auch deshalb, weil sie sonst als politische Vereine i.S.d. § 33 Vereinsgesetz 1867 zu qualifizieren wären, was im Widerspruch zu der faktischen Vernetzung der Vereine stünde.

${ }^{21}$ Knirsch führt dies auf jene Gehilfen zurück, die auf traditionelles Handwerk spezialisiert waren und sich weigerten, mit den Arbeitern aus der Industrie zu kooperieren oder ihr Handwerk zu modernisieren. Siehe KNIRSCH, Arbeiterbewegung 13.

22 Ebd. 19.

${ }^{23}$ Die Bewegung wird hier von mir bewusst als national sozialistisch und nicht als nationalsozialistisch bezeichnet. Dies deshalb, weil es sich um eindeutig sozialistische Forderungen handelt, die so auch in Parteiprogrammen der Sozialdemokraten $\mathrm{zu}$ finden sind (etwa im „Linzer Programm“ der Sozialdemokratischen Arbeiterpartei Österreich von 1926, nachzulesen in BERCHTOLD, Österreichische Parteiprogramme 18681966 247f.). Der Unterschied besteht darin, dass sich die Partei für die deutschen Arbeiter einsetzte und ein Nationalitätenkonflikt im Kern des Programmes zu finden ist. Noch finden sich ideologische Unterschiede zwischen den „Arbeiterparteien“ und der später von
}

Spaltung der - zeitweise durchaus am gleichen Strang ziehenden - Lager.

Der „Deutschnationale Verein“ löste sich bereits 1889 selbst auf. ${ }^{24}$ Grund dafür waren die vielen verschiedenen politischen Ansichten innerhalb der Deutschnationalen. Schönerer hatte es geschafft, sich mit beinahe allen namhaften und einflussreichen Vereinsmitgliedern $\mathrm{zu}$ zerstreiten. Die von ihm ins Leben gerufene Bewegung hätte damit wohl geendet, hätte sich nicht ein gewisser Personenkult um Schönerer selbst etabliert, der von verschiedenen kleineren Vereinen am Leben gehalten wurde. ${ }^{25}$ Diese 23 Vereine schlossen sich im Mai 1890 zum „,Waidhofener Verband“ zusammen und machten sich den „Kampf für das deutsche Volk" nach Vorlage Schönerers zur Aufgabe. ${ }^{26}$ Der Verband vertrat einen rassischen Antisemitismus. Man befasste sich bereits in einem Ausschuss mit der "Judenfrage" und entschied, dass Mitglieder „ihre arische Abstammung bis ins dritte Glied nachzuweisen“ hätten. ${ }^{27}$

Ebenfalls 1889 kam es zur Gründung des „Verbandes deutscher Gehilfen- und Arbeitervereinigungen Österreichs“, nach seinem Gründungsort

Hitler geführten NSDAP, unter anderem in der Parteistruktur, die kein Führerprinzip vorsah, sondern eine Wahl der Führung vorschrieb. Dass die Bewegung eindeutig antisemitische Forderungen vertrat, ist in meinen Augen deshalb kein Merkmal für eine Qualifikation als nationalsozialistisch, da die antisemitische Gesinnung weit verbreitet war. Es besteht natürlich genügend Spielraum für gegenteilige Ansichten. Je nachdem wie weit man die Definition von „nationalsozialistisch“ zieht, könnte man die Partei natürlich auch damals schon als nationalsozialistisch bezeichnen.

${ }^{24}$ WLADIKA, Hitlers Vätergeneration 228.

${ }^{25}$ Im Übrigen führte dies auch zu einer mehr oder weniger prominenten Stellung in jeder parteigeschichtlichen Schrift der späteren (deutschösterreichischen) Nationalsozialisten. Sogar in Hitlers "Mein Kampf wird er - neben Karl Lueger - erwähnt. Ausführlicher, insbesondere zum Einfluss Schönerers auf Hitler: HrTLER, Mein Kampf 309.

${ }^{26}$ WLADIKA, Hitlers Vätergeneration 239.

${ }^{27}$ Ebd. 
Mährisch Trübau [Moravská Třebová] „Mährisch-Trübauer Verband" genannt. Der Verband sollte die Gehilfenvereine trotz ihrer Differenzen einigen. Ihm gehörten 31 Vereine mit insgesamt 4.142 Mitgliedern an..$^{28}$ Auch dieser Verband war ideologisch dem politischen Konzept Schönerers nachempfunden. Erneut waren es aber interne Auseinandersetzungen zwischen Schönerer und seinen Weggefährten - in diesem Fall Karl Hermann Wolf, ${ }^{29}$ der eine eigene Partei gründete die das deutschnationale Lager spalteten. Dies führte schließlich zur Liquidierung des Mährisch-Trübauer Verbandes im November 1902. Bis zu seiner Auflösung war es dem Verband gelungen, sich auf 156 Vereine und 17.804 Mitglieder zu vergrößern. ${ }^{30}$

Auf derselben Konferenz, in der auch die Liquidierung des Mährisch-Trübauer Verbandes beschlossen wurde, entschied man sich auch, eine Partei zu gründen, die die deutschen Arbeiter in sich vereinen sollte: ${ }^{31}$ Die Deutsche Arbeiterpartei.

\footnotetext{
${ }^{28}$ CILLER, Vorläufer des Nationalsozialismus 26.

${ }^{29}$ Wolf erlangte wohl deshalb Bekanntheit, da er aus Protest gegen die Badenische Sprachenverordnung den Ministerpräsidenten Kasimir Felix Badeni so sehr beleidigte, dass dieser ihn zu einem Pistolenduell herausforderte. Badeni wurde am Arm verletzt, Wolf blieb unverletzt und wurde zum Helden seiner Anhänger. Siehe KORNAUTH, Graf Badeni als Ministerpräsident 93; HÖBELT, Kornblume und Kaiseradler 162.

${ }^{30} \mathrm{KNIRSCH}$, Arbeiterbewegung 23; für Details zu den Auseinandersetzungen, die sowohl persönlicher als auch politischer Natur waren: $\mathrm{KNIRSCH}$, Arbeiterbewegung $24 \mathrm{ff}$.

${ }^{31}$ HANKE, Die nationale Bewegung in Aussig 93.

32 WEBER, Adolf Hitler 7.

${ }^{33}$ WLADIKA, Hitlers Vätergeneration 524; ELSTE, NSDAP Kärnten 28; KNIRSCH, Arbeiterbewegung 30.

${ }^{34}$ ELSTE, NSDAP Kärnten 28; KNIRSCH, Arbeiterbewegung 30 .
}

\section{Von der Deutschen Arbeiterpar- tei zur DNSAP}

Die Gründung der Deutschen Arbeiterpartei, kurz DAP, wurde im Jahre 1903 auf einer Konferenz im böhmischen Aussig [Ústí nad Labem] beschlossen. ${ }^{32}$ Formal gegründet wurde die Partei ein Jahr später, im August 1904, in Trautenau [Trutnov]. ${ }^{33}$ Im Zuge dieses ersten ordentlichen Parteitages wurde auch das Parteiprogramm, genannt Trautenauer Programm, festgelegt. ${ }^{34}$ Politisch besann sich die Partei auf ihre nationalen Wurzeln zurück und widmete sich dem Kampf der deutschen Arbeiterschaft gegen die "fremdvölkischen" - also vor allem tschechischen - Arbeiter. Dies ist auch klar aus den Leitsätzen der Partei herauszulesen: „Die deutsche Arbeiterpartei erstrebt die Hebung und Befreiung der arbeitenden deutschen Volksschichten aus dem $\mathrm{Zu}-$ stande ihrer heutigen wirtschaftlichen, politischen und kulturellen Unterdrückung. ${ }^{\text {.335 Antise- }}$ mitische Forderungen waren nicht explizit genannt, verbargen sich allerdings im „Kampf gegen den fremdvölkischen Einfluss“ ${ }^{\prime 36}$

Der vor allem in Böhmen beliebten Partei gelang es 1911 sogar, drei Sitze im österreichischen Reichsrat zu erlangen. ${ }^{37}$ Eine erste Änderung erfuhr das Trautenauer Programm 1913, also kurz

\footnotetext{
${ }^{35} \mathrm{KNIRSCH}$, Arbeiterbewegung 29.

${ }^{36}$ GAWRECKI, Arbeiterpartei 33.

37 WLADIKA, Hitlers Vätergeneration 540. Die DAP kandidierte als Teil des Nationalverbands, der ein loser Zusammenschluss deutschfreiheitlicher Parteien war. Sie trat am 25. 6. 1912 aus dem Nationalverband aus und ihre Mitglieder traten als Hospitanten am 24. 10. 1912 wieder ein; siehe ADLGASSER, Zentralparlamente, Einleitung LXXX FN 259; BERNER, Vom Gesellenverein zum nationalen Sozialismus 184. Die Mitglieder waren Hans Knirsch (siehe ADLGASSER, Zentralparlamente 1, 593), Ferdinand Seidl (siehe ADLGASSER, Zentralparlamente 2, 1134) und Adam Fahrner (siehe ADLGASSER, Zentralparlamente 1, 271); WLADIKA, Hitlers Vätergeneration 561; SCHILLING, Geschichte des Nationalsozialismus 66; MASER, Frühgeschichte der NSDAP 241; KNIRSCH, Arbeiterbewegung 45; MALíř, Die Parteien in Mähren und Schlesien 778.
} 
vor dem Ersten Weltkrieg, auf dem Parteitag in Iglau [Jihlava]. Der wohl bedeutendste Unterschied zum Trautenauer Programm, das sich noch am Linzer Programm von 1882 orientierte, betrifft die Einstellung zu den Kronländern. Das Iglauer Programm forderte nach „völkischen Siedlungsgebieten" abgegrenzte Selbstverwaltungskörper und damit einhergehend eine vollständige Auflösung der Kronländer. ${ }^{38}$ Diese Änderungen wurden vor allem auf Betreiben von Rudolf Jung durchgeführt und von Walter Riehl ergänzt.

Der Beginn des Ersten Weltkrieges störte das Wachstum der Partei empfindlich. Zunächst bildete die Partei in den österreichischen und böhmischen Gebieten sowohl organisatorisch als auch personell eine Einheit. Bereits gegen Kriegsende, am 16. April 1918, fand ein Landesparteitag in Böhmen statt, auf dem die Umbenennung der DAP in Deutsche Nationalsozialistische Arbeiterpartei beschlossen wurde. Im Mai folgte ein entsprechender Beschluss auch am Parteitag in Wien. ${ }^{39}$ Nach dem Zusammenbruch der Österreichisch-Ungarischen Monarchie 1918 zerfiel die DNSAP 1918 in zwei Gruppierungen:40

1. Die in Deutschösterreich verbliebenen Anhänger der Partei bildeten die DNSAP Deutschösterreichs unter Führung von Walter Riehl.

\footnotetext{
${ }^{38}$ Originaltext und Ausführungen in $\mathrm{KNIRSCH}$, Arbeiterbewegung 32.

${ }^{39} \mathrm{Ob}$ diese nach Ländern getrennten Beschlüsse der Namensänderung aufgrund einer Vorahnung vom Zerfall der Monarchie, oder aber aufgrund der im Iglauer Programm festgehaltenen Trennung nach „,völkischen Siedlungsgebieten“ erfolgte, ist nicht klar. Jedenfalls schreibt Knirsch, dass „die Gründung der ,Nationalsozialistischen deutschen Arbeiterpartei' im Deutschen Reiche [...] ganz unabhängig von der Partei in Österreich erfolgte“, KNIRSCH, Arbeiterbewegung 57. Diese Behauptung ist insofern spannend, als kaum zwei Jahre später bereits die erste staatenübergreifende Tagung zwischen der deutschösterreichischen, der deutschen und der tschechoslowakischen Nationalsozialistischen Arbeiterpartei stattfand. Dies deutet darauf hin, dass die NSDAP in Deutschland keine historisch gemeinsamen Wurzeln mit ihrer Deutschnationalen Partei in der Monarchie gehabt hat. Das Werk ist
}

2. Die in der neu entstandenen Tschechoslowakei lebenden Parteianhänger organisierten sich unter ihrem Vorsitzenden Hans Knirsch. ${ }^{41}$

\section{Tschechoslowakei}

Während die österreichische Partei nie ein bedeutendes politisches Gewicht gewann, erlangte die DNSAP in der Ersten Tschechoslowakischen Republik eine gewisse Bedeutung. Die Partei konnte sich dabei im Gegensatz zur österreichischen DNSAP auf die Kernforderungen der DAP der Monarchie, nämlich die Verteidigung deutscher Arbeitsplätze gegen tschechische Arbeiter, stützen. ${ }^{42}$ Gemeinsam mit der bürgerlichen Deutschen Nationalpartei (DNP) unter Rudolf Lodgman von Auen vertrat sie jenen Teil der sudetendeutschen Bevölkerung, der der Gründung der Tschechoslowakei prinzipiell ablehnend gegenüber stand und den Anschluss der deutschsprachigen Gebiete an das Deutsche Reich oder an Deutschösterreich forderten. ${ }^{43}$ Alternativ verlangte die Partei ein „geschlossenes Siedlungsgebiet als gesicherten Lebensraum mit selbstgewählter Verfassung, Verwaltung und Landeswehr".44

1931 erschienen, Knirsch konnte damals wohl nicht absehen, welchen Einfluss Hitler bzw. die NSDAP auf seine tschechoslowakische Partei - und die europäische Geschichte - haben würde. Vielleicht hat Knirsch deshalb nicht versucht, eine künstliche Verbindung zwischen den Parteien zu konstruieren, wie dies so viele deutschösterreichische Nationalsozialisten in später erschienenen Werken versuchten.

${ }^{40}$ MASER, Frühgeschichte der NSDAP 243.

${ }^{41}$ KREBS, LeHMANN, Wir Sudetendeutsche 140.

${ }^{42} \mathrm{KNIRSCH}$, Arbeiterbewegung 68.

${ }^{43}$ BRANDSTÖTTER, Dr. Walter Riehl 146.

${ }^{44} \mathrm{KNIRSCH}$, Arbeiterbewegung 73; dabei berief sich die Partei auf das Pariser Minoritätenschutzgesetz. Siehe auch die Forderungen in KREBS, LehmanN, Wir Sudetendeutsche $18 \mathrm{ff}$. 
Bei den ersten Wahlen zum tschechoslowakischen Parlament im April 1920 ging die DNSAP ein Wahlbündnis mit der DNP unter dem Namen "Deutsche Wählergemeinschaft" ein. Dieses erreichte 5,3\% der Stimmen und 15 der 300 zu vergebenden Mandate; die DNSAP entsandte $5 \mathrm{Ab}$ geordnete ins Prager Abgeordnetenhaus; insgesamt entsandten die deutschen Parteien 72 Abgeordnete ins Parlament. 45

Im Zuge der politischen Stabilisierung der Tschechoslowakischen Republik im Verlauf der 20er Jahre konnte unter der sudetendeutschen Bevölkerung die kompromisslose Ablehnung des neuen Staates keine Popularität gewinnen. Der von den Parteien bei den nächsten Parlamentswahlen im November 1925 erhoffte Stimmenzuwachs blieb aus. Die DNP errang 3,4 \% der Stimmen (10 Mandate), die DNSAP 2,4\% (7 Mandate). ${ }^{46}$ Als Reaktion darauf traten die Vorsitzenden beider Parteien, Lodgman und Knirsch, zurück.

Im Jahre 1926 übernahmen Rudolf Jung und Hans Krebs die Parteiführung. Unter ihnen lehnte sich die Partei immer stärker an die NSDAP des Deutschen Reiches an. ${ }^{47}$ Bei den Parlamentswahlen im Oktober 1929 änderte das am Ergebnis der Partei wenig; sie errang nur 2,8 \% der Stimmen und 8 Mandate.

In den zunehmenden Wahlerfolgen der NSDAP im Deutschen Reich, der sich im Sudetenland besonders stark auswirkenden Weltwirtschaftskrise $^{48}$ und den damit wieder zunehmenden deutsch-nationalen Bestrebungen sah die tsche-

\footnotetext{
${ }^{45} \mathrm{KNIRSCH}$, Arbeiterbewegung 75; BRANDSTÖTTER, Dr. Walter Riehl 147.

${ }^{46}$ SLAPNICKA, Die böhmischen Länder 61.

${ }^{47}$ MASER, Frühgeschichte der NSDAP 238f.

48 Die deutschen Gebiete waren von der Arbeitslosigkeit stärker betroffen als die übrigen Gebiete der Tschechoslowakei; von 1.000 Einwohnern waren 90 arbeitslos. Ausführlicher zur Wirtschaftskrise: SLAPNICKA, Die böhmischen Länder 66.

${ }^{49}$ KREBS, LEHMANN, Wir Sudetendeutsche 158f.

50 SLAPNICKA, Die böhmischen Länder 77.
}

choslowakische Regierung eine ernste Bedrohung für ihren Staat. ${ }^{49}$ Die zunehmende paramilitärische Ausrichtung der tschechischen DNSAP mit der Schaffung eines "Verbunds Volkssport" nahmen die Behörden zum Anlass, die Partei zu verbieten. Diesem Verbot kam die DNSAP am 3. Oktober 1933 durch Selbstauflösung zuvor. ${ }^{50}$

\section{Österreich}

Die „Dismembration“ der Monarchie führte auch zum Zerbrechen der DAP. Schon beim Parteitag 1916 war man sich einig, dass die DAP umbenannt werden müsse, um einen größeren Wählerkreis anzusprechen. Man wollte die DAP in die „Deutschsoziale Arbeiterpartei Österreichs" umbenennen, um sich „die denkende Arbeiterschaft und die Arbeiteraristokratie zu holen, sodass den Sozialdemokraten nur mehr die urteilslose Masse blieb“. ${ }^{51}$ Hier merkte bereits Walter Riehl an, dass man nicht zu einer "Beamtenpartei“ verkommen wollte, die die Schutzarbeit für das deutsche Volk vernachlässigte. ${ }^{52}$ Riehl bestand darauf, dass der Parteiname schon den Inhalt der Parteileitsätze voll und ganz enthalten solle. Noch befand man sich auf ideologischer Wanderschaft. Im Mai 1918 benannte die Partei sich schließlich in „Deutsche Nationalsozialistische Arbeiterpartei“ um, wobei der Zusatz "sozialistisch" die Abgrenzung zu bürgerlichen Gruppen ausdrücken sollte. 1918/19 trat man dann mit einem neuen Parteiprogramm und dem Namen "Deutsche Nationalsozialistische Arbeiterpartei" (DNSAP) im Wahlkampf an. ${ }^{53}$

\footnotetext{
${ }^{51}$ WLADIKA, Hitlers Vätergeneration 578.

${ }^{52}$ BRANDSTÖTTER, Dr. Walter Riehl 121f.

${ }^{53}$ SCHILLING, Geschichte des Nationalsozialismus 7; KNIRSCH, Arbeiterbewegung 45. Höchstwahrscheinlich handelte es sich um einen (nicht politischen) Verein i.S.d. Vereinsgesetzes 1867, der sich zur Wahl als gleichnamige Wahlpartei formierte. Da die Wahlordnung zum Nationalrat die Parteien anerkennt und
} 
Der größte Teil der Mitglieder der Partei war seit jeher in den böhmischen Kronländern - der neu gebildeten Tschechoslowakei - beheimatet gewesen. Dies auch deswegen, weil der Nationalitätenkonflikt nur dort geführt werden konnte, wo auch Nationalitäten zusammentrafen. In den österreichischen Teilen der Monarchie hatte die Partei nie einen annähernd gleich großen Wählerkreis. Hinzu kam, dass in Deutschösterreich keine nationale Partei notwendig war, da das Land relativ „national einheitlich" war. ${ }^{54}$ Es galt daher, sich eine neue Klientel für die Wahl zu suchen. Ein altbewährtes Ziel waren auch hier wieder jene Tschechen, die teilweise schon lange vor dem Krieg nach Wien ausgewandert und dort geblieben waren. Diese „Post- und Bahnwenzel“ (da viele von ihnen bei der Post oder Bahn beschäftigt waren) sollten für die aus der Tschechoslowakei ausgewiesenen Deutschen Platz machen. ${ }^{55}$

Ein weiteres, vielversprechendes Angriffsziel für die Partei waren die Juden, von denen viele aus den ehemaligen Gebieten der Monarchie nach Wien zogen. Problematisch war jedoch, dass es einerseits viele kleine nationale Parteien gab und dass andererseits ihre Themen auch von größeren Parteien bedient wurden, so auch vom Parteiprogramm der Christlichsozialen, das an mehreren Stellen antisemitische Forderungen enthielt. ${ }^{56}$ Es fehlten der österreichischen DNSAP auch die finanziellen Mittel, um einen effektiven Wahlkampf zu führen. Eine Listenkoppelung blieb der Partei nicht erspart.

\section{Das Parteiprogramm der DNSAP ab 1918}

Im neuen Parteiprogramm blieben die meisten wirtschaftlichen und sozialen Forderungen und die „Forderungen der Arbeitnehmerschaft" aus dem Trautenauer Programm unangetastet, doch standen nun neue, rechte Leitsätze über den alten, linken sozialreformatorischen Forderungen. ${ }^{57}$ An erster Stelle stand die 1913 hinzugefügte "Hebung und Befreiung der arbeitenden deutschen Volksschichten aus wirtschaftlicher, politischer und geistiger Unterdrückung und ihre volle Gleichberechtigung auf sämtlichen Gebieten völkischen und staatlichen Lebens". ${ }^{58}$ Es sollte eine Besserung der Zustände durch „Zusammenfassung aller Schaffenden auf dem Boden des Volkstums“59 erreicht werden. Bereits hier waren die antisemitistischen Grundsätze erkennbar, die anscheinend untrennbar mit dem Nationalsozialismus verbunden sind. Denn nicht das Privateigentum an sich wurde als schädlich bezeichnet, sondern , alle Art arbeitslosen Einkommens, wie Grundrente und Geldzins und den [sic!] der Not des Nächsten abgepresste Wuchergewinn". ${ }^{60}$ Auch wenn anhand des Inhalts völlig klar war, wer vorrangig damit gemeint war, räumt das Programm durch explizite Nennung der Juden jegliche Zweifel am Antisemitismus der Partei aus dem Weg: „[Die Partei] bekämpft daher alle rückschrittlichen Bestrebungen, kirchlichen, adeligen und kapitalistischen Vorrechte und jeden fremdvölkischen Einfluß,

dentums und der jüdischen Presse verbündeten Parteien“ zu vertreten. Nachzulesen in BERCHTOLD, Österreichische Parteiprogramme 176.

57 WLADIKA, Hitlers Vätergeneration 580; MASER, Frühgeschichte der NSDAP 238.

${ }^{58}$ RIEHL, Endziel (1923) 15f.

${ }^{59}$ Ebd.; ELSTE, Kärntner NSDAP 28.

${ }^{60}$ RIEHL, Endziel (1923) $15 f$. ihnen an verschiedenen Stellen Rechte leitet Kraus daraus ihre Rechtssubjektivität ab. Siehe KRAUS, Die politische Partei als Rechtssubjekt 494f.

${ }^{55}$ Ebd. 125.

${ }^{56}$ So u.a. das Wahlmanifest der christlich-sozialen Reichspartei von 1907, welches erklärte, „,[dieses Programm] gegenüber den unter der Patronanz des Ju- 
vor allem aber die überwuchernde Macht des jüdisch-händlerischen Geistes auf allen Gebieten des öffentlichen Lebens." 61

Hinzu trat ein neuer, im Trautenauer Programm nicht vorhandener, Teil des Parteiprogrammes, der eng mit den ideologischen Grundsätzen der Partei in Verbindung stand. Dieser Programmpunkt umfasste die „Zusammenfassung des gesamten deutschen Siedlungsgebietes in Europa zum demokratischen, sozialen, Deutschen Reiche“ sowie den ",tatkräftigen Schutz aller von unserem Volke bewohnten aber von fremden Völkern beherrschten Gebiete“. ${ }^{62}$ Daraus ist auch das Bekenntnis der DNSAP zum "Anschluss" erkennbar. ${ }^{63}$ Auch die Ablehnung eines politischen Pluralismus zeigt sich in der Forderung, die Parteiherrschaft zu bekämpfen und über „einschneidende" Gesetze das Volk abstimmen zu lassen. ${ }^{64}$

Das im Februar 1920 von Adolf Hitler und Gottfried Feder entworfene Parteiprogramm der NSDAP weist viele Ähnlichkeiten mit dem Programm von Walter Riehl auf. Auch wenn in dem

\footnotetext{
${ }^{61}$ Ebd.; RIEHL, Endziel (1918) 9.

${ }^{62}$ RIEHL, Endziel (1923) 15f.; RIEHL, Endziel (1918) 11.

${ }^{63}$ BRANDSTÖTTER, Dr. Walter Riehl 131.

${ }^{64}$ RIEHL, Endziel (1923) 15f.

${ }^{65}$ Siehe Parteiprogramm in: BILLUNG, N.S.D.A.P. 145f;
}

HEIDEN, Geschichte des Nationalsozialismus 20f.

${ }^{66}$ SCHILLING, Geschichte des Nationalsozialismus 7; Wann genau sich die deutsche DAP in NSDAP umbenannte, ist nicht exakt feststellbar. Es soll jedoch Walter Riehl selbst gewesen sein, der vorschlug, dass die DAP "nationalsozialistisch" in ihren Namen aufnehmen solle. Siehe dazu: HitLeR, Mein Kampf 930 Rz 185. Gegenteiliger Ansicht ist, wie oben erwähnt, Knirsch, der ausdrücklich betont, dass die reichsdeutsche Nationalsozialistische Arbeiterpartei völlig unabhängig von der Deutschösterreichischen und der Tschechoslowakischen entstanden war. Ein regelmäßiger Kontakt zwischen den deutschen Arbeiterparteien zumindest ab 1920 ist aber gesichert belegt. Dies deckt sich auch mit der Praxis der Partei, die sowohl zwischen ihren lokalen Gruppierungen in der Monarchie als auch später zwischen der DNSAP Deutschösterreichs und jener der Tschechoslowakei regen Austausch pflegte. Namhafte Mitglieder wie etwa Riehl und Knirsch waren regelmäßig bei den Treffen der jeweils anderen Partei als von Hitler und Feder verfassten Programm der Antisemitismus noch um einiges radikaler ausgeprägt war, war der Kern der Forderungen gleich. ${ }^{65}$ Es war auch das Jahr 1920, in dem die erste gemeinsame, staatenübergreifende Tagung der deutschen NSDAP und der österreichischen DNSAP in Salzburg stattfand. ${ }^{66}$ Die Leitung der zwischenstaatlichen Kanzlei der Parteien wurde Walter Riehl übertragen. ${ }^{67}$ Noch hatte Walter Riehl die Parteiführung in Österreich inne, es sollte aber nicht lange dauern, bis Hitler immer mehr Einfluss auf die deutschösterreichische Partei erlangte. ${ }^{6}$

\section{Die Wahlen zur konstituierenden Nationalversammlung}

Die Wahl am 16. Februar 1919 brachte für die Partei eine herbe Enttäuschung. In den Wiener Wahlkreisen erreichte sie nur 0,7\% der Stimmen $^{69}$ und insgesamt kein einziges Mandat. ${ }^{70}$

Redner geladen. Siehe dazu: RosenKRANZ, SchulzGruppe 132f.; JAGSCHITZ, Nationalsozialistische Partei 233. Ein Flugblatt aus dem Jahr 1922, das zu einer Veranstaltung, bei der Hitler, Riehl und Jung geladen waren, einlud, findet sich im Wiener Stadt- und Landesarchiv: WStLA, Gauarchiv: Parteigeschichte, 2.7.1.11.1.A1.207.

${ }^{67}$ DeUERLEIN, Aufstieg der NSDAP 121.

${ }^{68}$ Obwohl sich die deutschösterreichische, die deutsche und die tschechoslowakische Partei in ihren Zielen und Grundsätzen sehr ähnlich waren, sei noch einmal darauf hingewiesen, dass die deutschösterreichische und die tschechoslowakische Partei ihre Führung wählten. Siehe dazu PAUlEY, Nationalsozialismus 40. Die mangelnde Bereitschaft, Hitlers Führerprinzip umzusetzen, war es auch, die zur Auseinandersetzung zwischen Riehl - und später Schulz - und Hitler und damit zur Spaltung der Partei führte.

${ }^{69}$ RosenKRANZ, Schulz-Gruppe 128.

${ }^{70}$ https://bmi.gv.at/412/Nationalratswahlen/files/NRW_1919.pdf (3. 9. 2019). 
Dies wurde von der Partei als äußerst enttäuschend empfunden. Als Reaktion auf das schlechte Ergebnis begann die DNSAP, die Parteipresse zu vermehren und ihre Organisationsarbeit $\mathrm{zu}$ intensivieren. ${ }^{71}$

Bei den Landtagswahlen 1919 erlangte die Partei in Salzburg zwei Mandate und im Wahlkreis Wien-Innen-Ost (1., 3., 4. Bezirk) ein Mandat für den niederösterreichischen Landtag, in den man Walter Riehl entsendete. Riehl forderte dort die Aufhebung des Mieterschutzes für Nichtdeutsche (v.a. „Ostjuden"), um Wohnungen für "Arier" frei zu machen, und die Einstellung der Ausgabe von Lebensmittelkarten an Juden sowie die Entlassung aller „Fremdvölkischen“, die im Staatsdienst tätig waren. ${ }^{72}$

Gleichzeitig kam es zur Gründung von Ortsvereinen der DNSAP und zur Gründung des „Deutschösterreichischen Schutzvereines Antisemitenbund“, der sich der „Befreiung aus der jüdischen Herrschaft" verschrieben hatte. ${ }^{73}$ Hauptthema waren die jüdischen Flüchtlinge aus der Bukowina und Galizien, die wegen der Kriegsverwüstung und der antisemitischen Ausschreitungen in ihren Ländern nach Wien kamen. Das Thema beschäftigte allerdings nicht nur die Deutschnationalen und die Christlichsozialen, sondern auch die Sozialdemokraten. ${ }^{74}$ Die Anti-

\footnotetext{
${ }^{71}$ BRANDSTÖTTER, Dr. Walter Riehl 149.

72 Ebd. 150f.

${ }^{73}$ In Kärnten „Väterländischer Schutzbund“ genannt, welcher eine Keimzelle für die spätere SA war. ELSTE, Kärntner NSDAP 33

${ }^{74}$ Siehe z.B. das Parteiprogamm der Wiener Christlichsozialen von 1919, welches den „Ausgleich zwischen Interessen der verschiedenen Bevölkerungsschichten auf christlicher, deutscher und antisemitischer Grundlage“ zu vertreten versprach. Nachzulesen in BERCHTOLD, Österreichische Parteiprogramme 1868-1966 364.

${ }^{75}$ LiCHTBLAU, Antisemitismus 455

${ }^{76}$ RosenKRANZ, Schulz Gruppe 130; LichtBLAU, Antisemitismus 455 .

77 Ein Flugblatt, betitelt als „Die Wahlkomödie ist vorüber", mit welchem die Partei erklärte „diesen
}

semitismuswelle gipfelte schließlich in einem Erlass des sozialdemokratischen niederösterreichischen Landeshauptmannes Albert Sever, der eine Ausweisung aller zugezogenen Juden verlangte. ${ }^{75}$ Daran ist deutlich erkennbar, dass sich bis auf wenige Parteien alle an der Judenhetze beteiligten, mit der man den Juden die Schuld am verlorenen Krieg, an der Wohnungsnot und der Lebensmittelknappheit zuschrieb. ${ }^{76}$

\section{Nationalratswahlen 1923}

Die Frage, ob man für die Nationalratswahlen 1923 eine Einheitsliste mit der Großdeutschen Volkspartei bilden sollte, spaltete die Partei. Die Mehrheit entschied sich gegen eine Teilnahme an den Wahlen. ${ }^{77}$ Walter Riehl, der sich für eine Wahlgemeinschaft mit den Großdeutschen ausgesprochen hatte, wurde für diese Haltung stark kritisiert. ${ }^{78}$ Statt zu einer Beteiligung an den Wahlen entschloss man sich, den „Väterländischen Schutzbund" auszubauen und so das Führerprinzip auch auf Österreich auszuweiten. Riehl, der mit dieser Entscheidung nicht einverstanden war, aber die Partei durch seine Gegenstimme nicht spalten wollte, legte sein Amt nieder. ${ }^{79}$ Seine Geschäfte wurden von Karl Schulz weitergeführt und Riehl selbst wurde von allen Parteigeschäften beurlaubt. ${ }^{80}$

Schwindel nicht mitgemacht [zu haben] " findet sich im Wiener Stadt- und Landesarchiv: WStLA, Gauarchiv: Parteigeschichte, 2.7.1.11.1.A1.207.

78 SCHILLING, Geschichte des Nationalsozialismus 79f; Riehl orientierte sich dabei an der sudetendeutschen Bruderpartei, die es durch Listenkoppelung mit den Deutschnationalen in das Prager Parlament geschafft hatte.

${ }^{79}$ BRANDSTÖTTER, Dr. Walter Riehl 197; ELSTE, Kärntner NSDAP 36.

${ }^{80}$ Die kommenden Jahre Riehls waren von politischer Bedeutungslosigkeit und Distanzierung von der DNSAP geprägt. Mit seinen Anhängern gründete er den „Deutschsozialen Verein für Österreich“. 1930 trat Riehl der Hitler-Bewegung bei, wurde jedoch zwei Jahre später ausgeschlossen. 1933 distanzierte er sich 
Das Wahlergebnis zeigte, dass Riehl mit seiner Haltung gegenüber der Einheitsliste recht gehabt hatte. Die Partei verzeichnete einen deutlichen Verlust an Stimmen, was zu Meinungsverschiedenheiten innerhalb der Partei führte. Grund für die ideologische Spaltung war, dass viele der jüngeren Parteimitglieder einen anderen Zugang zur Politik hatten. Das Leben der jüngeren Parteimitglieder war nicht mehr bestimmt von dem Kampf um „deutsche“ Arbeitsplätze in einem tschechisch dominierten Umfeld, sondern von der Erniedrigung durch den verlorenen Krieg und die Pariser Vororteverträge. ${ }^{81}$ Die jungen Parteimitglieder glaubten nicht daran, dass Riehl und seine Generation in der Lage waren, den Traum von einem „alldeutschen Reich“ erfüllen zu können. Schulz, der Nachfolger Riehls, war ein Gewerkschafter, der die Partei wieder zu ihrem Ursprung führen wollte und daher zwangsläufig mit Hitler in Konflikt geraten musste. ${ }^{82}$

\section{Vom Hitlerputsch bis zum Ende der Partei}

Bei der fünften gemeinsamen Tagung der deutschen und (deutsch-)österreichischen Nationalsozialisten im August 1923 hatte Hitler seine Haltung gegenüber der österreichischen Partei klar zu erkennen gegeben. Der Grund für die ablehnende Haltung Hitlers gegenüber der österreichischen Partei ist wohl in der unterschiedlichen Parteienstruktur zu finden; während die deutsche NSDAP das Führerprinzip schon früh parteiintern etabliert hatte, wurde die Parteiführung

erneut von den nationalsozialistischen Terrorakten. Nach dem Anschluss 1938 versuchte er sich Hitler zu unterstellen, wurde jedoch verhaftet. Nur eine Intervention eines hohen Parteifunktionärs verhinderte seine Überführung in ein Konzentrationslager; siehe JAGSCHITZ, Nationalsozialistische Partei 233f. Dokumente über die Tätigkeit des Deutschsozialen Vereins, zum Teil von Riehl selbst verfasst, finden sich im Wiener Stadt- und Landesarchiv: WStLA, Gauarchiv: Parteigeschichte, 2.7.1.11.1.A1.207. der DNSAP noch von ihren Mitgliedern gewählt. Über die deutschen Nationalsozialisten hatte Hitler bereits nahezu diktatorische Befugnisse - und er war nicht gewillt, einen Widerspruch zu seiner Politik durch die wesentlich kleinere österreichische Partei zu dulden. ${ }^{83}$

Kurz nach Riehls Rücktritt fügte der gescheiterte Putsch in München der österreichischen Nationalsozialistischen Partei den nächsten schweren Schlag zu. Man sammelte Geld für die Verletzten und die Hinterbliebenen der Verstorbenen und half, Teilnehmer des Putsches, unter anderem Hermann Göring, nach Österreich zu schmuggeln. ${ }^{84}$ Die Stärke der Partei nahm nach dem gescheiterten Putsch rapide ab. Sie konnte sich davon erst nach der Neugründung der deutschen NSDAP im Februar 1925 erholen. Mit der Neugründung erhielten auch die österreichischen Nationalsozialisten neuen Zulauf. Aber auch der 14. Zionistische Weltkongress, der im August 1925 in Wien stattfand und den die Partei zum Anlass für die Organisation von Protestversammlungen und Tumulte nutzte, verhalf der Partei zu neuer Anhängerschaft. ${ }^{85}$ Gleichzeitig spaltete der Weltkongress aber die jungen Anhänger erneut von der alten Führung, da letztere nicht auf die Straße gehen wollte. Schulz versuchte die jungen Mitglieder seiner Partei von gewalttätigen Ausschreitungen abzuhalten. Die von Hitlers diktatorischer Führung und radikaler Rhetorik begeisterte Jugend der DNSAP war mit dem langsamen „Fortschritt" der österreichischen Partei immer unzufriedener. Obwohl Schulz im Februar 1926 einen letzten Versuch unternahm, mit dem er die Partei einen wollte,

\footnotetext{
${ }^{81}$ BILLUNG, N.S.D.A.P. 59.

82 RosenKRANZ, Schulz-Gruppe 178.

${ }^{83}$ PAUley, Nationalsozialismus 45.

84 Ebd. 47; JAGSCHITZ, Nationalsozialistische Partei 233.

${ }^{85}$ BRANDSTÖTTER, Dr. Walter Riehl 227; LiCHTBLAU, Antisemitismus 456.
} 
mussten im Mai 1926 über 200 Mitglieder, nämlich jene, die sich von der DNSAP eine Parteipolitik im Sinne und Stil Hitlers wünschten, aus der deutschösterreichischen Partei ausgeschlossen werden. Die Ausgeschlossenen beantworteten ihren Ausschluss mit der Gründung des sogenannten „Hitlervereins", der sich direkt Hitler unterstellte. ${ }^{86}$

Diese - erneute - Spaltung innerhalb der DNSAP sorgt für einige Verwirrungen und Verwechslungen, weshalb sie hier noch einmal kurz im Überblick behandelt werden soll. Die DNSAP Deutschösterreich spaltete sich zuerst 1923, als Riehl seine Parteifunktionen zurücklegte und einen eigenen Verein gründete. ${ }^{87}$ Bezeichnet wird dieses Fragment der Partei oft als „RiehlGruppe“. Bei den nach dem Rücktritt Riehls in der DNSAP verbliebenen Mitgliedern handelt es sich um die "Schulz-Gruppe“, benannt nach dem Nachfolger Riehls, Karl Schulz. Es stellte sich für Schulz bald heraus, dass Hitler von ihm nur blinden Gehorsam forderte und die Überzeugungen und Strukturen der DNSAP nicht berücksichtigte. Jener Teil der DNSAP, der von Hitlers Politik begeistert war und eine radikalere nationalsozialistische Vorgehensweise der deutschösterreichischen Partei forderte, war es, der von Schulz im Mai 1926 aus der Partei aufgrund seiner Radikalität ausgeschlossen wurde. Ihrer politischen Einstellung entsprechend gründeten jene Mitglieder den „Nationalsozialistischen Verein (Hitlerbewegung) “ 88 - auch „Hitlerverein“ genannt. 89 Jegliche Versuche in den Jahren 1927 und 1929, die Partei wieder zu vereinen, blieben erfolglos. Die beiden Gruppierungen bekämpften einander bis zur Auflösung der "Schulz-Gruppe“ 1934,

\footnotetext{
86 JAGSCHITZ, Nationalsozialistische Partei 233, 235.

${ }^{87}$ Der „Freundschaftbund Dr. Walter Riehl“ - später „Deutschsozialer Verein für Österreich“ genannt, bestand hauptsächlich aus den Anhängern Riehls. Er wurde am 3. 6. 1924 gegründet. Siehe dazu: BRANDSTÖTTER, Dr. Walter Riehl $216 f$.

${ }^{88}$ ROSENKRANZ, Schulz-Gruppe 226.
}

wobei die Hitlerbewegung seit Hitlers Wahlerfolg in Deutschland 1930 die Oberhand hatte. Die Hitlerbewegung wurde 1933 nach einer Reihe nationalsozialistischer Terrorakte von der Regierung Dollfuß aufgelöst. ${ }^{90}$

\section{Die DNSAP - eine faschistische Partei?}

Dass es einen „roten Faden" der ideologischen Entwicklung von Georg von Schönerer und den Gehilfenvereinen bis zu der in (Deutsch-) Österreich tätigen DNSAP gibt, und dieser Partei somit eine "Brückenfunktion" zwischen dem Deutschnationalismus in der Habsburgermonarchie einerseits, und der NSDAP Adolf Hitlers andererseits zukommt, unterliegt keinem Zweifel. Wesentlich schwieriger ist die Frage zu beantworten, ob die DNSAP selbst eine faschistische Partei war, bzw. wo sich die ideologische Wandlung von den deutschnationalen, antisemitischen Vereinen $\mathrm{zu}$ einer nationalsozialistischen, faschistischen Partei vollzog. Nennt man als primäre Merkmale der österreichischen Nationalsozialisten, dass sie den Anschluss befürworteten und antisemitisch gesinnt waren, so müsste man wohl sehr vielen Parteien (Deutsch-) Österreichs eine nationalsozialistische Gesinnung unterstellen. Der Anschlusswunsch wurde u.a. aus wirtschaftlichen Gründen von einer breiten Bevölkerungsgruppe geteilt und kann für sich allein nicht ausschlaggebend sein. Aber auch der Antisemitismus war weit verbreitet - was

\footnotetext{
${ }^{89}$ Aufgrund des Zeitpunktes ihrer Gründung ist davon auszugehen, dass es sich sowohl beim „Deutschnationalen-Verein“ als auch beim „Hitlerverein“ um Vereine i.S.d. Vereinsgesetz handelte.

${ }^{90}$ Siehe GARSCHA, Nationalsozialisten in Österreich 103f. Die Auflösung erfolgte mit Verordnung vom 19. 6. 1933, BGBl. 240/1933.
} 
man nicht oft genug betonen kann. ${ }^{91}$ Diese religiöse und rassische Diskriminierung ist ein tief in der Geschichte der Monarchie und Republik verankerter schwarzer Fleck, der nicht verharmlost werden sollte, indem man ihn als eine Ideologie darstellt, die allein dem Nationalsozialismus zukam und mit diesem auch wieder verschwand.

Wo beginnt also der Faschismus? Hält man die Implementierung eines Führerprinzips für das wichtigste Merkmal, so muss man wohl zu dem Schluss kommen, dass die DNSAP keine faschistische Partei war. Denn die Führung der DNSAP wurde - im Gegensatz zu Hitlers NSDAP - gewählt. Stellt man auf den Willen der Partei ab, durch Gewalt an die Macht zu kommen, so entstehen die ersten faschistischen Züge der DNSAP um das Jahr 1925, als die jungen Parteimitglieder beginnen, sich ausdrücklich für terroristische Akte auszusprechen. Orientiert man sich an einem rassischen Antisemitismus, so wird man spätestens beim Waidhofener Verband (1890) fündig. Dort verlangte man bereits einen, die Nürnberger Rassegesetze gleichsam vorwegnehmenden, Ariernachweis „,bis ins dritte Glied“ für Parteimitglieder. Zuletzt darf auch nicht vergessen werden, dass das Parteiprogramm der DNSAP von 1918 viele Gemeinsamkeiten mit dem Parteiprogramm der NSDAP von 1920 hat.

Aufgrund der schwer fassbaren Definition des Faschismus ist eine klare Zuordnung der DNSAP nicht möglich. Vielmehr sollte eine differenzierte Betrachtung herangezogen werden. Einzig die ab 1926 in Österreich tätige Hitlerbewegung kann aufgrund ihrer Gewaltbereitschaft und ihrer Unterordnung unter Hitler eindeutig als faschistische, nationalsozialistische Partei bezeichnet werden. $\mathrm{Ob}$ auch ihre deutschnationalen, antisemitischen Vorgänger als faschistische, oder als

\footnotetext{
${ }^{91}$ Ein prominentes Beispiel hierfür wäre wohl der Mediziner Theodor Billroth, der, scheinbar völlig unreflektiert, den rassischen Antisemitismus in seinem Buch „Über das Lehren und Lernen der medizinischen Wissenschaft an den Universitäten“ vertrat: „daß ein
}

"gewöhnliche" Parteien in einem habituell anschlussfreundlichen bzw. deutschnationalen und antisemitischen politischen Umfeld gesehen werden können, ist letztlich eine Definitionsfrage.

\section{Korrespondenz:}

\author{
Mag. Carmen KLEINZSIG \\ Universität Wien \\ Institut für Rechts- und Verfassungsgeschichte \\ Schottenbastei 10-16 \\ 1010 Wien \\ ORCID-Nr. 0000-0002-5850-4530
}

\section{Abkürzungen:}

Siehe das allgemeine Abkürzungsverzeichnis:

[http://www.rechtsgeschichte.at/media/abk.pdf]

\section{Literatur:}

Franz ADLGASSER, Die Mitglieder der österreichischen Zentralparlamente 1848-1918. Konstituierender Reichstag 1848-1849, Reichsrat 1861-1918, 2 Bde. (Wien 2014).

Klaus BERCHTOLD, Österreichische Parteiprogramme 1868-1966 (Wien 1967).

Alfred BERNER, Vom Gesellenverein zum nationalen Sozialismus. Betrachtungen über die Entwicklung der nationalsozialistischen Bewegung (Troppau 1930).

R. BILLUNG, N.S.D.A.P. Die Geschichte einer Bewegung (München 1931).

Rudolf BRANDSTÖTTER, Dr. Walter Riehl und die Geschichte der nationalsozialistischen Bewegung in Österreich (ungedr. phil. Diss., Univ. Wien 1969).

Alois CILLER, Vorläufer des Nationalsozialismus. Geschichte und Entwicklung der nationalen Arbeiterbewegung im deutschen Grenzland (Wien 1932).

Ernst DeuERLEIN, Der Aufstieg der NSDAP in Augenzeugenberichten (Düsseldorf 1968).

Jude, ebensowenig wie ein Perser, oder ein Franzose oder ein Neuseeländer, oder Afrikaner, je ein Deutscher werden kann [...]" siehe hierzu ausführlich: WLADIKA, Hitlers Vätergeneration 45. 
Alfred ELSTE, Stationen der Kärntner NSDAP 19191933: Von der völkischen Protestpartei zur aufstrebenden Massenbewegung, in: DERS., Dirk HäNISCH (Hgg.), Auf dem Weg zur Macht. Beiträge zur Geschichte der NSDAP in Kärnten von 1918 bis 1938 (Wien 1997) 27-62.

Dan GAWRECKI, Německá dělnická Strana 1904-1919. K pronikání buržoazního nacionalismu do dělnického hnutí [Die deutsche Arbeiterpartei 19041918. Zum Einbruch des bourgeoisen Nationalismus in die Arbeiterbewegung], in: Slezský sbornik [Schlesischer Almanach] (Opava 1971) 29-41, 8189.

Winfred R. GARSCHA, Nationalsozialisten in Österreich 1933-1938, in: Emmerich TÁlOS, Wolfgang NEUGEBAUER (Hgg.), Austrofaschismus. Politik - Ökonomie - Kultur 1933-1938 (Wien 2005) 100-20.

Alfred HANKE, Die nationale Bewegung in Aussig von 1848-1914. Ein Beitrag zur Geschichte des deutschen Nationalismus in den Sudentenländern (Prag 1943).

Adolf Hitler, Mein Kampf. Eine kritische Edition, Bd. 1, hg. v. Christian HARTMANN u.a. (MünchenBerlin 2016).

Konrad HeIDEN, Geschichte des Nationalsozialismus. Die Karriere einer Idee (Berlin 1932).

Lothar HöBELT, Kornblume und Kaiseradler. Die deutschfreiheitlichen Parteien Altösterreichs 18821918 (Wien 1993).

Gerhard JAGsCHITZ, Die Nationalsozialistische Partei, in: Emmerich TÁlos u.a. (Hgg.), Handbuch des politischen Systems Österreichs. Erste Republik 19181933 (Wien 1995) 231-244.

Hans KNIRSCH, Aus der Geschichte der deutschen nationalsozialistischen Arbeiterbewegung Alt-Österreich und der Tschechoslowakei (Aussig 1931).

Friedrich KORNAUTH, Graf Badeni als Ministerpräsident (phil. Diss., Univ. Wien 1949).

Gaston J. KRAUS, Die politische Partei als Rechtssubjekt: politische Partei und Wahlpartei, in: JBl (1929) 493-498.

Hans KREBS, Emil LEHMANN, Wir Sudentendeutsche! (Berlin 1938).

Arthur LENHOFF, Die politische Partei als Rechtssubjekt, in: JBl (1929) 250-251.

Albert LichtBlAU, Antisemitismus - Rahmenbedingungen und Wirkungen auf das Zusammenleben von Juden und Nichtjuden, in: Emmerich TÁLOS u.a. (Hgg.), Handbuch des politischen Systems Österreichs. Erste Republik 1918-1933 (Wien 1995) 454-471.

Jiří MALíř, Die Parteien in Mähren und Schlesien und ihre Vereine, in: Helmut RuMPLER, Peter URBANITSCH (Hgg.), Die Habsburger Monarchie 18481918, Band VIII/1 (Wien 2006) 705-803.

Jiří MALíř (Hg.), Politické strany: vývoj politických stran a hnutí v českých zemích a Československu 1861-2004. I. díl, Období 1861-1938 [Politische Parteien: Entwicklung der politischen Parteien und Bewegungen in den böhmischen Ländern und der Tschechoslowakei 1861-2004. Teil I, Zeitraum 18611938] (Brno 2005).

Werner MASER, Die Frühgeschichte der NSDAP. Hitlers Weg bis 1924 (Frankfurt-Bonn 1965).

Ernst MAYRHOFER, Handbuch für den politischen Verwaltungsdienst in den im Reichsrathe vertretenen Königreichen und Ländern mit besonderer Berücksichtigung der diesen Ländern gemeinsamen Gesetze und Verordnungen, Bd. II (Wien ${ }^{51896) . ~}$

Bruce F. PAULEY, Der Weg in den Nationalsozialismus. Ursprünge und Entwicklung in Österreich (Wien 1988).

Jiři POKORNÝ, Vereine und Parteien in Böhmen, in: Helmut RuMPLER, Peter URBANITSCH (Hgg.), Die Habsburger Monarchie 1848-1918, Bd. VIII/1 (Wien 2006) 609-703.

Ilse REITER-ZATLOUKAL, Christiane ROTHLÄNDER, Pia SCHÖLNBERGER (Hgg.), Österreich 1933-1938. Interdisziplinäre Annäherungen an das Dollfuß-/ Schuschnigg-Regime (Wien 2012).

Walter RIEHL, Unser Endziel. Eine Flugschrift für den deutschen Nationalsozialismus (Wien 1918, 51923).

Hannes RosENKRANZ, Die "Schulz-Gruppe“ der NSDAP (phil. Diss., Univ. Wien 2004).

Alexander SchILLING, Dr. Walter Riehl und die Geschichte des Nationalsozialismus (Berlin 1933).

Helmut SLAPNICKA, Die böhmischen Länder und die Slowakei 1919-1945, in: Karl BosL (Hg.), Handbuch der Geschichte der böhmischen Länder, Bd. IV: Der Tschechoslowakische Staat im Zeitalter der modernen Massendemokratie und Diktatur (Stuttgart 1970) 2-151.

Thomas WeBER, Wie Adolf Hitler zum Nazi wurde. Vom unpolitischen Soldaten zum Autor von „Mein Kampf" (Berlin 2016).

Michael WLADIKA, Hitlers Vätergeneration. Die Ursprünge des Nationalsozialismus in der k.u.k. Monarchie (Wien 2005). 